\title{
Evaluation of Front Morphological Development of Reactive Solute Transport Using Behavior Diagrams
}

\author{
Jui-Sheng Chen ${ }^{1}$, Yuan-Yao Chang ${ }^{2}$, and Chen-Wuing Liu ${ }^{2,3, *}$ \\ ${ }^{1}$ Graduate Institute of Applied Geology, National Central University, Chung-Li, Taiwan, ROC \\ ${ }^{2}$ Department of Bioenvironmental Systems Engineering, National Taiwan University, Taipei 106, Taiwan, ROC \\ ${ }^{3}$ Institute of Hydrotech, National Taiwan University, Taipei 106, Taiwan, ROC
}

Received 9 June 2008, accepted 7 November 2008

\begin{abstract}
While flowing through porous medium, groundwater flow dissolves minerals thereby increasing medium porosity and ultimately permeability. Reactive fluid flows preferentially into highly permeable zones, which are therefore dissolved most rapidly, producing a further preferential permeability enhancement. Accordingly, slight non-uniformities present in porous medium can be amplified and lead to fingering reaction fronts. The objective of this study is to investigate dissolution-induced porosity changes on reaction front morphology in homogeneous porous medium with two non-uniformities. Four controlling parameters, including upstream pressure gradient, reaction rate constant, non-uniformities spacing and non-uniformity strength ratio are comprehensively considered. By using a modified version of the numerical code, NSPCRT, to conduct a series of numerical simulations, front behavior diagrams are constructed to illustrate the morphologies of reaction fronts under various combinations of these four factors. Simulation results indicate that the two non-uniformities are inhibited into a planar front under low upstream pressure gradient, merge into a single-fingering front under intermediate upstream pressure gradient, or grow into a double-fingers front under high upstream pressure gradient. Moreover, the two non-uniformities tend to develop into a double-fingering front as the non-uniformity strength ratio increases from 0.2 to 1.0 , and merge into a single-fingering front while the non-uniformity strength ratio increases from 1.0 to 1.8. When the reaction rate constant is small, the two non-uniformities merge into a single front. Reaction rate constant significantly affects front advancing velocity. The front advancing velocity decreases with the reaction rate constant. Based on these results, front behavior diagrams which define the morphologies of the reaction fronts for these four parameters are constructed. Moreover, non-uniformity strength ratio and reaction rate constant are identified as two important factors that govern the interaction of dissolution and solute transport in groundwater systems.
\end{abstract}

Key words: Porosity, Kinetic dissolution reaction, Non-uniformity strength ratio, Reaction rate constant, Front behavior diagram Citation: Chen, J. S., Y. Y. Chang, and C. W. Liu, 2009: Evaluation of front morphological development of reactive solute transport using behavior diagrams. Terr. Atmos. Ocean. Sci., 20, 853-862, doi: 10.3319/TAO.2008.11.07.01(Hy)

\section{INTRODUCTION}

When groundwater flows through porous media of the subsurface, various reactions such as dissolution and precipitation occur between groundwater and mineral grains. Media porosity and species concentration are also subsequently modified. Changes in porosity affect the groundwater flow pattern. If porosity in some region of the porous medium is slightly higher than elsewhere (we refer to it as a non-uniformity herein), groundwater flow in this non-uni-

\footnotetext{
* Corresponding author

E-mail:lcw@gwater.agec.ntu.edu.tw
}

formity accelerates and dissolution reaction rates are raised. A rise in the dissolution reaction rate producesa higher porosity channel, thereby resulting in a feedback complex system. The above phenomenon often occurs in geological systems (Chen and Ortoleva 1990; Ortoleva 1994; Fredd and Fogler 1998; Lasaga 1998). Consequently, the interaction between groundwater flow and the solid matrix of geological media has received much attention (Xu and Pruess 2001; Chen and Liu 2002; Emmanuel and Berkowitz 2005; Zhao 2008). Several extensive studies, including field and laboratory experiments, and numerical model developments and 
simulations have been conducted to provide further understanding of water-rock interaction. Chadam et al. (1986) developed a two dimensional mathematical model, coupling a set of the governing partial differential equations of groundwater flow, species transport and porosity change induced by dissolution to investigate the dissolution-induced morphologies of reaction fronts. The simulation results indicate the upstream pressure gradient governs the shapes of reaction fronts. Under a high upstream pressure gradient and long flow channel width, a single non-uniformity may develop into a single-fingering, double-fingering or even multiple-fingering front (Chadam et al. 1986; Ortoleva et al. 1987a, b; Chen and Ortoleva 1990; Daccord et al. 1993; Renard et al. 1998; Xu and Pruess 2001; Chen and Liu 2002; Emmanuel and Berkowitz 2005; Singurindy and Berkowitz 2005; Li et al. 2006). Chen and Liu (2004) used the numerical model, NSPCRT (Chen and Liu 2002), to conduct numerical modeling of reaction front instability, temporal aquifer porosity, and species concentration evolution for groundwater flow in a homogeneous medium with two local non-uniformities of equal strength. They found that planar, single-fingering or double-fingering fronts can develop under various upstream pressure gradients. The shape of the fronts is dependent upon the distance between the local non-uniformities and the size of the pressure gradient. Simulation results indicated that a planar front develops under a low upstream pressure gradient, and a single-fingering or double-fingering front can emerge under a high upstream pressure gradient. A front behavior diagram defining the morphology of the reaction fronts was also constructed.

In natural geological media, the strengths of the two non-uniformities are likely to be unequal. In addition, this earlier work does not consider the reaction rate constant though it is also a controlling factor during reactive transport. Therefore, besides the upstream pressure gradient and non-uniformity spacing, the reaction rate constant and nonuniformity strength ratio (the ratio of the strength of the first non-uniformity to that of the second), should be included for studying reaction front behavior. The objective of this study is to evaluate the evolution of reaction front morphology induced by dissolution. Four parameters: upstream pressure gradient, reaction rate constant, non-uniformity spacing and non-uniformity strength ratio are comprehensively considered to investigate the evolution of reaction front morphology. The computer model, NSPCRT (Chen and Liu 2002) is adopted and modified to execute a series of numerical simulations under various combinations of these four parameters. According to the simulation results, front behavior diagrams are constructed presenting the morphologies of the different reaction fronts.

\section{MATHEMATICAL MODEL}

The dynamics of changes in media porosity, perme- ability, and species concentration caused by mineral dissolution reactions in groundwater systems can be formulated by a set of coupled nonlinear partial differentiation equations. In this study, we modify the two-dimensional numerical model, NSPCRT (Chen and Liu 2002) to simulate the above phenomenon. The modifications made to NSPCRT are described in section 3 . The governing equations for porosity changes affected by kinetic dissolution reactions, the conservation of groundwater flow, and species transport are described below.

\subsection{Porosity Changes Due to Kinetic Chemical Dissolution}

Change in pore volume occurs as a result of the reduction in volume of the solid phase induced by mineral dissolution. The relationship between porosity change and kinetic chemical dissolution can be obtained by invoking conservation of total volume in the porous media. NSCPRT assumes that porous media consists of pores and soluble and insoluble grains. The mineral grains are cubic in shape and occupy a given volume with the number of mineral grains remaining constant after dissolution. Moreover, single solid components such as calcite in the porous medium and single species such as calcium or carbonate ions in the groundwater are considered with dissolution reactions following the first-order kinetics equation. The porosity change due to kinetic chemical dissolution is thus expressed as:

$\frac{\partial \phi}{\partial \mathrm{t}}=-\Gamma^{1 / 3}\left(\phi_{\mathrm{f}}-\phi\right)^{2 / 3}\left(\mathrm{C}-\mathrm{C}_{\mathrm{sat}}\right)$

where $\phi$ is the porosity (dimensionless); $\Gamma$ denotes the dissolution reaction rate constant $\left(\mathrm{M}^{-1} \mathrm{~L}^{4} \mathrm{~T}^{-1}\right) ; \mathrm{n}$ represents the number of mineral grains per unit volume $\left(\mathrm{L}^{-3}\right)$; $\mathrm{C}$ is species concentration $\left(\mathrm{ML}^{-3}\right) ; \phi_{\mathrm{f}}$ is the final porosity after a complete dissolution of soluble grains (dimensionless), and $\mathrm{C}_{\mathrm{sat}}$ is the saturated species concentration in groundwater $\left(\mathrm{ML}^{-3}\right)$.

In order to evaluate how the reaction rate constant affects groundwater/porous media reactions and species transport processes, we assume:

$\Gamma=\alpha \Gamma^{\prime}$

where $\Gamma^{\prime}$ is the dissolution reaction rate constant of soluble porous media at $25^{\circ} \mathrm{C}\left(\mathrm{M}^{-1} \mathrm{~L}^{4} \mathrm{~T}^{-1}\right)$, and $\alpha$ denotes a parameter for convenient treatment of dissolution rate constant changes (dimensionless).

Equation (1) can then be rewritten as:

$\frac{\partial \phi}{\partial \mathrm{t}}=-\alpha \Gamma^{\prime} \mathrm{n}^{1 / 3}\left(\phi_{\mathrm{f}}-\phi\right)^{2 / 3}\left(\mathrm{C}-\mathrm{C}_{\mathrm{sat}}\right)$ 
In Eq. (3), the dissolution reaction rate constant changes with $\alpha$.

\subsection{Conservation of Groundwater Flow}

The governing equation of groundwater flow is derived by applying mass conservation of groundwater and Darcy's law, and is written as:

$\nabla \cdot[\phi \mathrm{K}(\phi) \nabla \mathrm{P}]=\frac{\partial \phi}{\partial \mathrm{t}}$

where $\mathrm{P}$ denotes the fluid pressure $\left(\mathrm{ML}^{-1} \mathrm{~T}^{-2}\right)$, and $\mathrm{K}(\phi)$ is the intrinsic permeability divided by the product of the water viscosity and porosity $\left(\mathrm{M}^{-1} \mathrm{~L}^{3} \mathrm{~T}\right)$. A modified form of the Fair-Hatch relation (Chen and Liu 2002) is employed herein to characterize the dependence of permeability on porosity (Bear 1972):

$$
\mathrm{K}(\phi)=\frac{\phi^{3}}{\mathrm{~J} \mu \theta^{2}\left[\left(1-\phi_{\mathrm{f}}\right)^{2 / 3}+\mathrm{n}^{1 / 3}\left(\phi_{\mathrm{f}}-\phi\right)^{2 / 3}\right]^{2}}
$$

where $J$ denotes a packing factor $(\sim 5)$ (dimensionless); $\mu$ is the fluid viscosity $\left(\mathrm{ML}^{-1} \mathrm{~T}^{-1}\right)$; and $\theta$ is a geometric factor (dimensionless). It should be noted that elevation head is neglected in Eq. (4). However, Eq. (4) can be used to describe, for example, two-dimensional horizontal groundwater flow without vertical head gradient. Moreover, the developed model, NSCPRT, can be readily modified to simulate three-dimensional groundwater flow by including elevation head into Eq. (4).

\subsection{Conservation of Species Transport}

The model assumes that groundwater flow velocity is slow and dispersion effect resulting from the spatial variability in the pore water velocity is excluded. Besides advection and diffusion processes governing solute transport, dissolution reactions also cause a mass transfer of solids into respective aqueous phases, thus raising species concentrations in an aqueous fluid. A partial differential equation derived via mass conservation of solutes is expressed in the following:

$\nabla \cdot[\phi \mathrm{D}(\phi) \nabla \mathrm{C}+\phi \mathrm{CK}(\phi) \nabla \mathrm{P}]+\rho_{\mathrm{s}} \frac{\partial \phi}{\partial \mathrm{t}}=\frac{\partial(\phi \mathrm{C})}{\partial \mathrm{t}}$

where $\mathrm{D}(\phi)$ denotes the porosity dependent diffusion coefficient $\left(\mathrm{L}^{2} \mathrm{~T}^{-1}\right)$, and $\rho_{\mathrm{s}}$ represents the density of soluble grains $\left(\mathrm{ML}^{-3}\right)$. The common phenomenological relation for
$\mathrm{D}(\phi)$ is (Bear 1972; Lerman1979):

$\mathrm{D}(\phi)=\mathrm{D}_{\mathrm{i}} \phi^{\mathrm{M}} \quad\left(\frac{3}{2}<\mathrm{M}<\frac{5}{2}\right)$

where $D_{i}$ is the solute diffusion coefficient $\left(\mathrm{L}^{2} \mathrm{~T}^{-1}\right)$. The first, second and third terms on the left-hand side of Eq. (5) represent the diffusion flux, advection flux and source due to mineral dissolution, respectively.

Equations (3), (4), and (5) are the governing equations of the model, and are all nonlinear partial differential equations. Porosity $(\phi)$, solute concentration $(\mathrm{C})$, and fluid pressure $(\mathrm{P})$ are primary variables, and are all function of spatial and temporal variables. Theoretically, we can solve the set of coupled Eqs. (3), (4), and (5), then illustrate the processes of porosity and solute concentration change with time and space. To simplify the coupled equations, Eqs. (3), (4), and (5) are thus non-dimensionalized. First, dimensionless time, $\tau$, is defined by:

$\tau=\varepsilon \Gamma^{\prime} n^{1 / 3} \mathrm{C}_{\mathrm{sat}} \mathrm{t}$

where $\varepsilon=\mathrm{C}_{\mathrm{sat}} / \rho_{\mathrm{s}}$.

The dimensionless solute concentration, $\gamma$, the dimensionless fluid pressure, $\overline{\mathrm{P}}$, the dimensionless diffusion coefficient, $d$, and the dimensionless permeability, $\lambda$, are respectively defined by:

$$
\begin{aligned}
& \gamma=\frac{\mathrm{C}}{\mathrm{C}_{\mathrm{sat}}} \\
& \overline{\mathrm{P}}=\mathrm{P} \frac{\mathrm{K}\left(\phi_{\mathrm{f}}\right)}{\mathrm{D}\left(\phi_{\mathrm{f}}\right)}
\end{aligned}
$$

$\mathrm{d}(\phi)=\frac{\phi \mathrm{D}(\phi)}{\mathrm{D}\left(\phi_{\mathrm{f}}\right)}$

$\lambda(\phi)=\frac{\phi \mathrm{K}(\phi)}{\mathrm{K}\left(\phi_{\mathrm{f}}\right)}$

Dimensionless solute concentration now ranges from $0.0\left(\gamma_{\min }\right)$ to $1.0\left(\gamma_{\max }\right)$. Spatial variables are also non-dimensionalized as:

$\bar{x}=x\left[\frac{\Gamma^{\prime} n^{1 / 3} C_{s a t}}{D\left(\phi_{\mathrm{f}}\right)}\right]^{1 / 2}$

$\bar{y}=y\left[\frac{\Gamma^{\prime} \mathbf{n}^{1 / 3} C_{\text {sat }}}{D\left(\phi_{f}\right)}\right]^{1 / 2}$ 
Based on the definition of Eqs. (11) and (12), the dimensionless gradient of spatial variables can be written as:

$\bar{\nabla}=\left[\frac{\Gamma \mathrm{n}^{1 / 3} \mathrm{C}_{\mathrm{sat}}}{\mathrm{D}\left(\phi_{\mathrm{f}}\right)}\right]^{-1 / 2} \cdot \nabla$

where $\bar{\nabla}=\frac{\partial}{\partial \overline{\mathbf{x}}} \overrightarrow{\mathbf{i}}+\frac{\partial}{\partial \overline{\mathbf{y}}} \overrightarrow{\mathrm{j}}$.

Incorporating Eqs. (6) - (13) into Eqs. (3), (4), and (5) and rearranging them in dimensionless form gives:

$\varepsilon \frac{\partial \phi}{\partial \tau}=-\alpha\left(\phi_{\mathrm{f}}-\phi\right)^{2 / 3}(\gamma-1)$

$\varepsilon \frac{\partial \phi}{\partial \tau}=\bar{\nabla} \cdot(\lambda \bar{\nabla} \overline{\mathrm{P}})$

$\bar{\nabla} \cdot[\mathrm{d}(\phi) \bar{\nabla} \gamma+\lambda(\phi) \gamma \bar{\nabla} \overline{\mathrm{P}}]+\frac{\partial \phi}{\partial \tau}=\varepsilon \frac{\partial(\phi \gamma)}{\partial \tau}$

Equations (14) - (16) can be solved numerically by the sequential iteration approach (SIA) where the equations for reaction-induced porosity changes and conservations of groundwater flow and solute transport are solved sequentially. The detailed steps of numerically solving Eqs. (14) (16) using the SIA were demonstrated by Chen and Liu (2002)

\section{NUMERICAL SIMULATION}

This study considers a two dimensional rectangular domain with the length of $\mathrm{L}_{\bar{x}}$ (dimensionless length) and the width of $\mathrm{L}_{\bar{y}}$ (dimensionless length), as shown in Fig. 1 . Groundwater flows from left to right. The inlet (left-hand side boundary) condition for dimensionless pressure is prescribed as:

$\frac{\partial \overline{\mathrm{P}}(\overline{\mathrm{x}}, \overline{\mathrm{y}}=0, \tau)}{\partial \overline{\mathrm{x}}}=-\mathrm{P}_{\mathrm{f}}$

where $\mathrm{P}_{\mathrm{f}}$ denotes an upstream pressure gradient applied at the inlet boundary. The upstream pressure gradient has a major influence on the dissolution reaction and solute transport (Chadam et al. 1986; Chen and Liu 2002, 2004).

Only the net pressure gradient across the region of interest is important since the fluid density is assigned to be a constant. Accordingly, a referenced pressure is prescribed at the outlet (right-hand) boundary as:

$\overline{\mathrm{P}}\left(\overline{\mathrm{x}}=\mathrm{L}_{\overline{\mathrm{x}}}, \overline{\mathrm{y}}, \tau\right)$
No flow conditions are used for the upper and lower boundaries and expressed as:

$\frac{\partial \overline{\mathrm{P}}(\overline{\mathrm{x}}, \overline{\mathrm{y}}=0, \tau)}{\partial \overline{\mathrm{y}}}$

$\frac{\partial \overline{\mathrm{P}}\left(\overline{\mathrm{x}}, \overline{\mathrm{y}}=\overline{\mathrm{L}_{\overline{\mathrm{y}}}}, \tau\right)}{\partial \overline{\mathrm{y}}}$

The boundary condition on the left-hand side for dimensionless concentration is prescribed to be zero:

$\gamma(\bar{x}=0, \bar{y}, \tau)=0$

The right, upper, and lower boundary conditions for dimensionless concentrations are, respectively, prescribed as:

$\frac{\partial \gamma\left(\overline{\mathrm{x}}=\mathrm{L}_{\overline{\mathrm{x}}}, \overline{\mathrm{y}}, \tau\right)}{\partial \overline{\mathrm{y}}}$

$\frac{\partial \gamma(\bar{x}, \bar{y}=0, \tau)}{\partial \bar{y}}$

$\frac{\partial \gamma\left(\overline{\mathrm{x}}, \overline{\mathrm{y}}=\mathrm{L}_{\overline{\mathrm{y}}}, \tau\right)}{\partial \overline{\mathrm{y}}}$

Assuming the system is homogeneous except for two unequal strength non-uniformities existing with non-uniformity centers located at the inlet boundary. The non-uniformities are zones of higher porosity and permeability located at the left boundary of the system, as shown in Fig. 2. The initial conditions for the dimensionless porosity and concentration are set as follows:

$\phi(\bar{x}, \bar{y}, 0)=\phi_{0}+\left(\phi_{\mathrm{f}}-\phi_{0}\right)\left(\mathrm{e}^{-\xi_{1}}+\mathrm{e}^{-\xi_{2}}\right)$

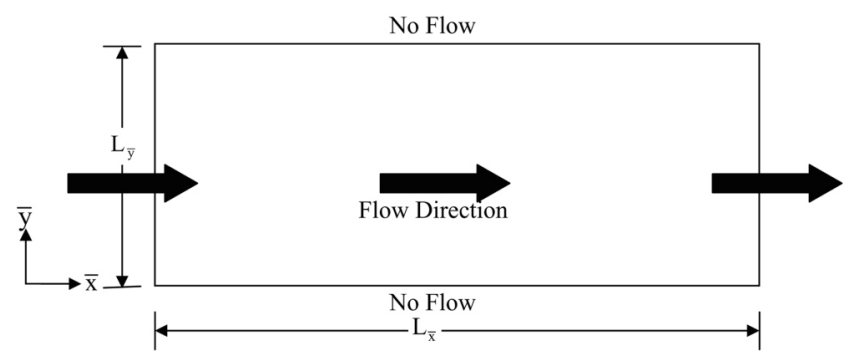

Fig. 1. Domain for numerical simulation. 
(a) Planar front

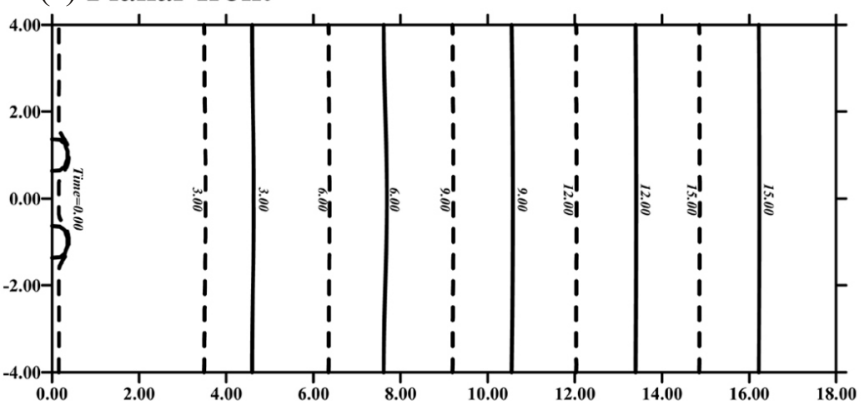

(c) Double-fingering front

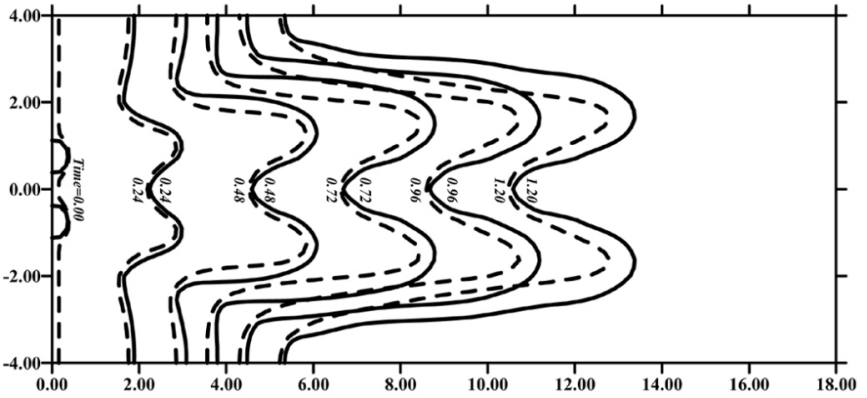

(b) Single-fingering front

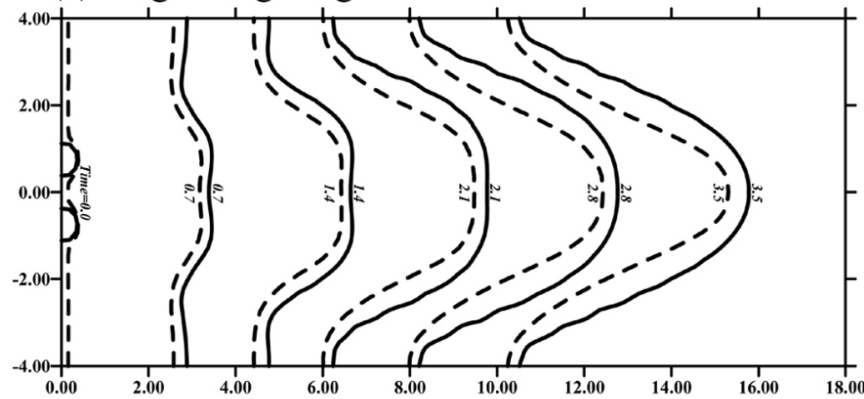

(d) Transition zone

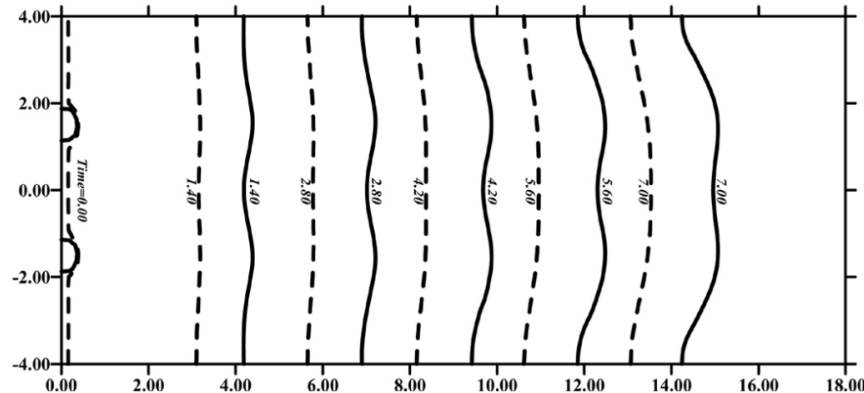

Fig. 2. Four types of reaction front morphology. Solid line: aquifer porosity, dashed line: species concentration.

$\gamma(\overline{\mathrm{x}}, \overline{\mathrm{y}}, 0)=\left(1-\mathrm{e}^{-5 \overline{\mathrm{x}}}\right)\left(1-\mathrm{e}^{-\xi_{1}}-\mathrm{e}^{-\xi_{2}}\right)$

with

$\xi_{1}(\bar{x}, \bar{y})=S S \times\left[\bar{x}^{4}+\left(\bar{y}-L_{1}\right)^{4}\right] /\left(w_{\bar{y}}\right)^{4}$

$\xi_{2}(\bar{x}, \bar{y})=\left[\bar{x}^{4}+\left(\bar{y}-L_{2}\right)^{4}\right] /\left(w L_{\bar{y}}\right)^{4}$

where $L_{i}(i=1,2)$ represent the locations of centers of first and second non-uniformities, $w$ denotes an initial perturbation parameter which is used to perturb the initial porosity and dimensionless concentration, and SS denotes the nonuniformity strength ratio of the first non-uniformity to the second non-uniformity.

The initial conditions for the dimensionless pressure are set to be:

$\overline{\mathrm{P}}(\overline{\mathrm{x}}, \overline{\mathrm{y}}, \tau=0)=0$

To execute the numerical simulation it is necessary to give the values of all the parameters in the set of governing equations and boundary conditions. In this study, we give the input parameters following our previous studies (Chen and Liu 2002, 2004). The input parameters used in previous studies were assumed and successfully used to demonstrate the morphological evolution of a dissolution fingering front in a fluid-saturated porous medium. The inputs were: initial porosity $\phi_{0}=0.1$; final porosity $\phi_{\mathrm{f}}=0.2$; dimensionless length $\mathrm{L}_{\overline{\mathrm{x}}}=18$; dimensionless width $\mathrm{L}_{\overline{\mathrm{y}}}=8$; initial perturbation parameter $\mathrm{w}=0.1$, and $\overline{\mathrm{x}}$ and $\overline{\mathrm{y}}$ grid spacing are $0.2 \times 0.2$.

To examine whether the construction of front behavior diagram using a 30 -set simulation (coarse simulation) is adequately to represent the evolution of front morphology, we carried out a comparison between the coarse and detailed simulations. The non-uniformity strength ratio, SS, is set to be 1.0 in both coarse and detailed simulations. In the coarse simulation, the upstream pressure gradient changes from 0.0 to 5.0 with an increment of 1.0 and non-uniformity spacing varies from 1.0 to 5.0 with an increment of 1.0. Whereas in the detailed simulation, upstream pressure gradient, $\mathrm{P}_{\mathrm{f}}$, is set from 0.00 to 5.0 with a 0.25 increment. Non-uniformity spacing is set from 0.25 to 5.00 with an increment of 0.25 and $\alpha$ is set to be 1.0. A total of 441 sets of simulations are executed.

To investigate the effects of the non-uniformity strength ratio and dissolution reaction rate constant on the development of front morphology, two cases are considered as follows:

\subsection{Case I: Different Strength Ratios (SS)}

In this case, non-uniformity strength ratio, SS, is set 
from 0.2 to 2.0 with an increment of 0.2. Upstream pressure gradient, $\mathrm{P}_{\mathrm{f}}$, is set from 0.0 to 5.0 with an increment of 1.0. Initial two perturbations spacing is set from 1.0 to 5.0 with an increment of 1.0 and $\alpha$ is set to be 1.0. There are a total of $300(10 \times 6 \times 5)$ simulation sets.

\subsection{Case II: Different Dissolution Reaction Rate Constant $(\alpha)$}

In this case, non-uniformity strength, SS is fixed at 1.0. Upstream pressure gradient, $\mathrm{P}_{\mathrm{f}}$, varies from 0.0 to 5.0 with an increment of 1.0. Non-uniformity spacing changes from 1.0 to 5.0 with a 1.0 increment and $\alpha$ is set from 0.2 to 1.0 with an increment of 0.2 . There are a total $150(5 \times 6 \times 5)$ simulation sets.

Each simulation ends when the porosity is greater than 0.2 or the dimensionless concentration is greater than 1.0 at the right-hand boundary $\left(\overline{\mathrm{x}}=\mathrm{L}_{\overline{\mathrm{x}}}, \overline{\mathrm{y}}=\overline{\mathrm{y}}\right)$ or the dimensionless time arrives at the maximum simulation time $(\tau=10.0)$. Front morphology is determined based on reaction front shapes at the time the simulation ends.

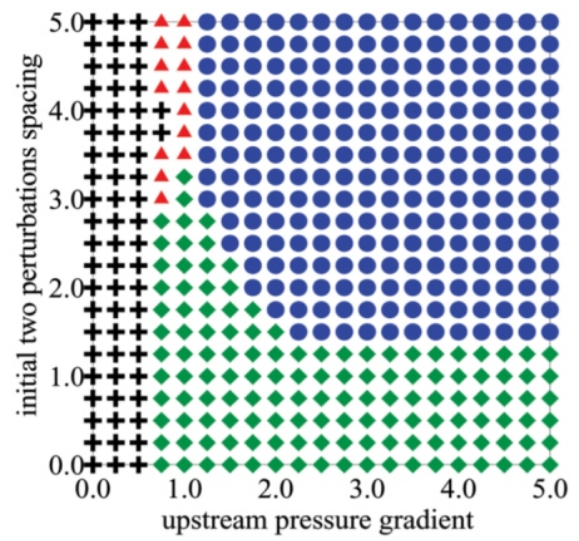

(a) Detail simulated results

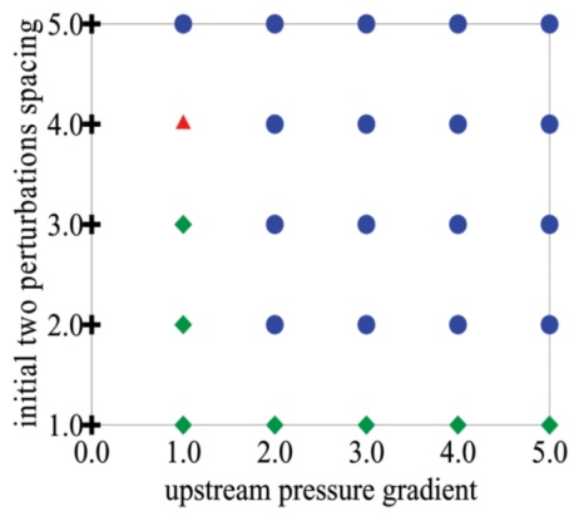

(c) Coarse simulated result

\section{RESULTS AND DISCUSSION}

The simulation results showed that with two non-uniformities, four types of reaction-front shapes can develop (see Fig. 2). A planar front (Fig. 2a) denotes that the contours of porosity and dimensionless concentration are constants along the $\bar{y}$ direction. A single-fingering front (Fig. $2 b$ ) denotes that two non-uniformities merge into a single finger. A double-fingering front (Fig. 2c) represents that the two non-uniformities grow into two clear fingers. If the front shape cannot be clearly judged, we herein classify it as in a transition zone (Fig. 2d). As shown in Fig. 2, the evolution of front morphology of porosity contours and dimensionless concentration contours are similar, we herein construct the behavior diagram of the front morphology only based on dimensionless porosity contours.

\subsection{Verification of Behavior Diagram by Detailed Simulation}

Figure 3 represents the front behavior diagram con-

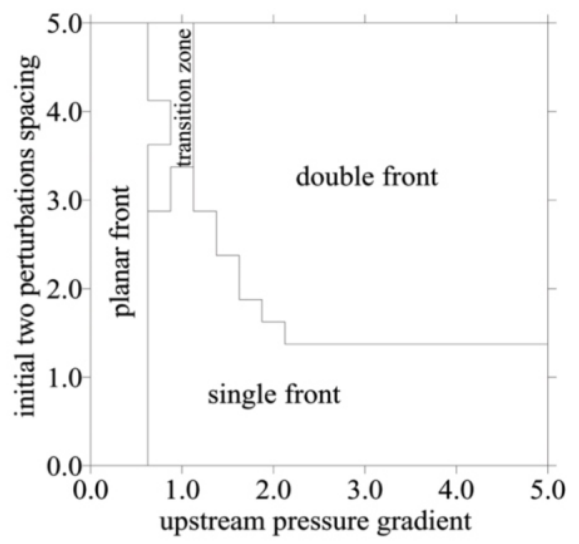

(b) Detailed simulated behavior diagram

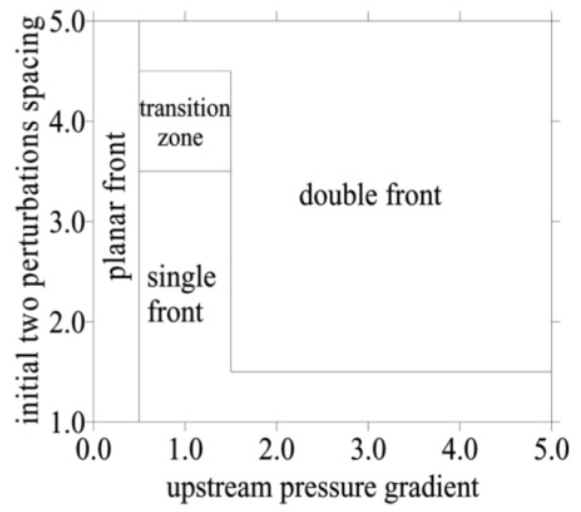

(d) Coarse simulated behavior diagram

Fig. 3. Comparison of coarse and detailed simulated results and behavior diagrams of SS $=1.0$ and $\alpha=1.0$. $\mathbf{\uparrow}$ denotes planar front, $\Delta$ denotes transition zone, $\bullet$ denotes single front, and $\bullet$ denotes double front. 
structed from the detailed and coarse simulations. A planar front is located at the zone where the upstream pressure gradient is less than 0.5 and 0.625 for coarse (Fig. 3d) and detailed simulations (Fig. 3b), respectively. The location and size of the planar front zone are similar. The zones of the single-fingering and double-fingering fronts are also similar. Therefore, the front behavior diagram constructed from 30 sets of coarse simulation can adequately represent the front morphology with the upstream pressure gradient varying from 0.0 to 5.0 and non-uniformity spacing changing from 1.0 to 5.0. The behavior diagram of the same conditions established by Chen and Liu (2004) is similar to that of this study. The only difference is that the transition zone is approximately located between upstream pressure gradients of 0.6 to 1.0 , and is not affected by non-uniformity spacing. In the detailed simulation, the transition zone is approximately located between upstream pressure gradients of 0.75 to 1.0 and non-uniformities spacing greater than 2.8 . The reason is that the increments of the upstream pressure gra- dient and spacing in the simulation of Chen and Liu (2004) are 0.5 whereas the increment in the detailed simulation of this study is 0.25 , which yields a more refined result.

\subsection{Behavior Diagrams of Different SS and $\alpha$}

\subsubsection{Case I: Change of Perturbation Strength Factor (SS)}

Figure 4 shows the simulated behavior diagrams for non-uniformity strength ratios varying from 0.2 to 2.0 with an increment of 0.2. Planar front areas are all located at low upstream pressure gradient $\left(\mathrm{P}_{\mathrm{f}}<0.3\right)$. Double-fingering front areas are located at high upstream pressure gradient and non-uniformity spacing greater than 1.5. Single-fingering front areas are located in between planar front and double fronts. As the two non-uniformities have non-equal strengths, such as when $\mathrm{SS}=0.2$, the porosity at the large non-uniformity strength zone is higher than that at the smaller strength zone resulting in high groundwater velocity (a) $\mathrm{SS}=0.2, \quad \alpha=1.0$

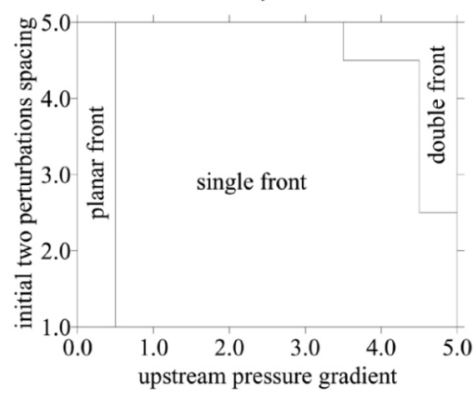

(d) $\mathrm{SS}=0.8, \quad \alpha=1.0$

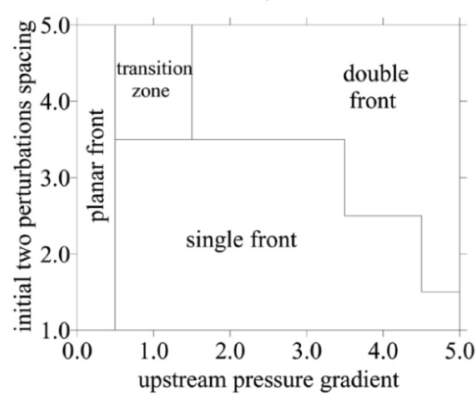

(g) $\mathrm{SS}=1.4, \quad \alpha=1.0$

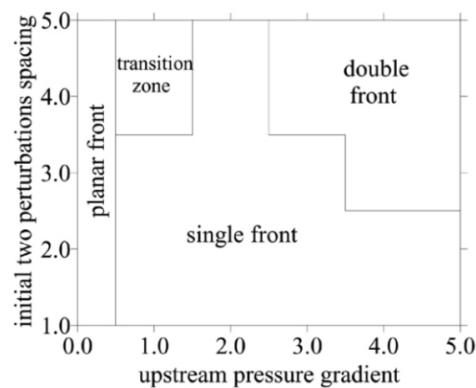

(b) $\mathrm{SS}=0.4, \quad \alpha=1.0$

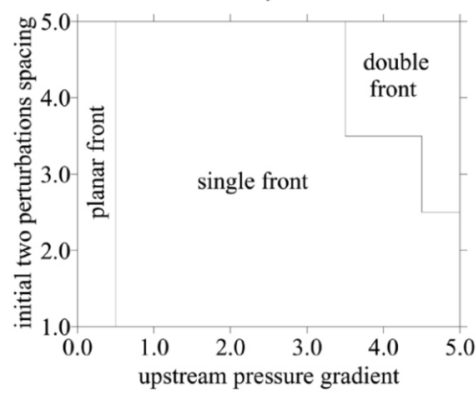

(e) $\mathrm{SS}=1.0, \quad \alpha=1.0$

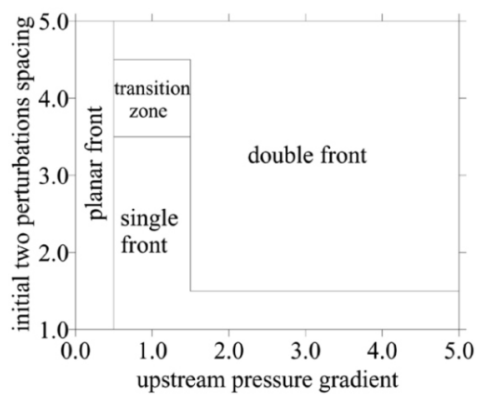

(h) $\mathrm{SS}=1.6, \alpha=1.0$

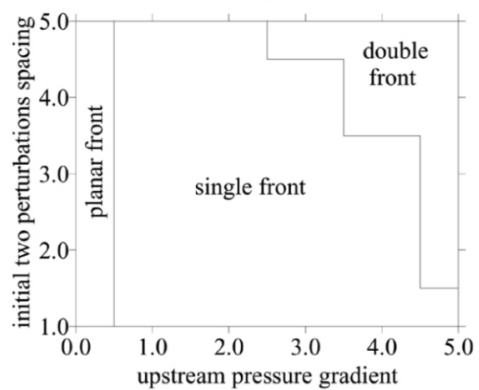

(c) $\mathrm{SS}=0.6, \alpha=1.0$

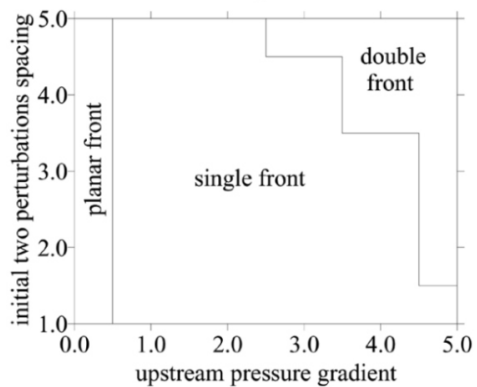

(f) $\mathrm{SS}=1.2, \quad \alpha=1.0$

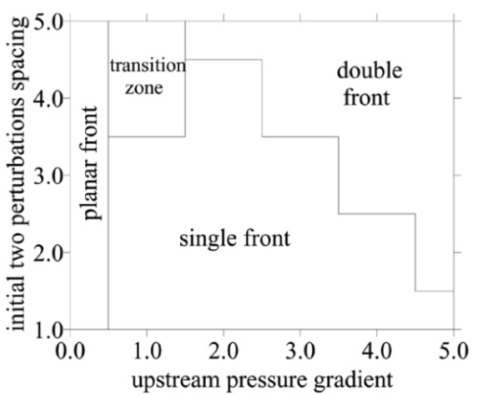

(i) $\mathrm{SS}=1.8, \alpha=1.0$

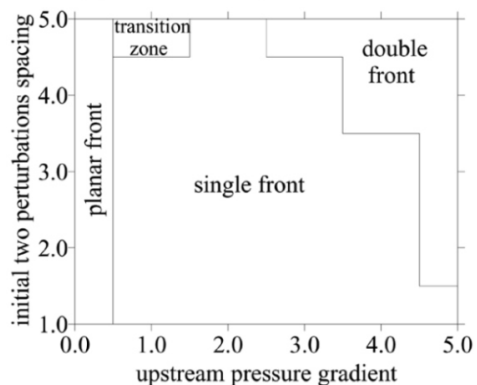

Fig. 4. Behavior diagrams for different strength ratios (SS). 
developing in the large non-uniformity strength zone. Advective transport due to the fast groundwater velocity, first lowers the solute concentration, then accelerates the kinetic dissolution reaction rate and enlarges the influential area of the front. Thereby, the front at the larger non-uniformity strength zone propagates faster and extends into a larger area than that at the smaller non-uniformity strength zone. As the two fronts continuously develop, at a certain critical time, the rate at which porosity increases between the two non-uniformities may become equal to that at the smaller strength zone such that the two non-uniformities then merge into a singlefingering front. As the non-uniformity strength ratio increases from 0.2 to 1.0 , the areas of the single-fingering front decrease, and those of the double-fingering front increase; the optimum condition for developing a double-fingering front is at $\mathrm{SS}=1.0$. A planar front is formed when advection due to a low upstream pressure gradient is not sufficient to lower the solute concentration resulting from kinetic dissolution reactions and diffusion transport. Thus, the kinetic dissolution reaction rate reduces and then slows down the porosity change in the zones of the two non-uniformities. As the porosity changes at the two non-uniformities become the same as the ambient zones, a planar front steadily develops. These results agree with previous studies (Chadam et al. 1986; Chen and Ortoleva 1990; Chen and Liu 2002).

As the non-uniformity strength ratio increases from 1.0 to 1.8 , the two non-uniformities are apt to merge into a single-fingering front and the area of the double-fingering front gradually decreases as shown in Figs. $4 \mathrm{f}-\mathrm{i}$.

\subsubsection{Case II: Changes in Dissolution Reaction Rate Constant $(\alpha)$}

Figure 5 represents the front behavior diagram for $\alpha$ varying from 0.2 to 1.0 with a 0.2 interval. The areas of the double-fingering front in the front behavior diagrams constructed with $\alpha$ of 0.2 and 0.4 are smaller than those obtained with $\alpha$ of $0.6,0.8$, and 1.0. Low reaction rate constants cause a slow increase in porosity in the zone of the initial two perturbations. Although advection transport in the zones of the two non-uniformities is high, slow porosity change rates further reduce front advancing velocity. As the porosity change rate of the two non-uniformities lowers to the same as that of the zone between the two non-uniformities, the two non-uniformities merge into a single-fingering front.

Changes of $\alpha$ slightly affect the front behavior diagrams, but significantly affect the front advancing velocity. Figures $6 \mathrm{a}-\mathrm{c}$ represent advection, diffusion and resultant flux for: a non-uniformity strength ratio of 1.0 , non-uniformity spacing of 2.0, $\alpha$ of 0.6 , and upstream pressure gradient of 2.0. Figures $7 \mathrm{a}-\mathrm{c}$ represent advection, diffusion and resultant flux for: non-uniformity strength ratio of 1.0, nonuniformity spacing of 2.0, $\alpha$ of 1.0, and upstream pressure gradient of 2.0. In Figs. 6a, b and 7a, b, both advection flux tends to focus toward the tip of the front while the diffusion flux tends to pull back the front tip. The advection flux of $\alpha=1.0$ is greater than that of $\alpha=0.6$ and the diffusion flux of $\alpha=1.0$ is greater than that of $\alpha=0.6$, the resultant of both advection and diffusion fluxes of $\alpha=1.0$ is still higher than (a) $\mathrm{SS}=1.0, \quad \alpha=0.2$

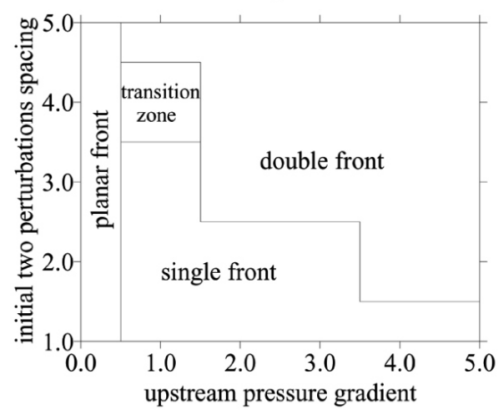

(d) $\mathrm{SS}=1.0, \quad \alpha=0.8$

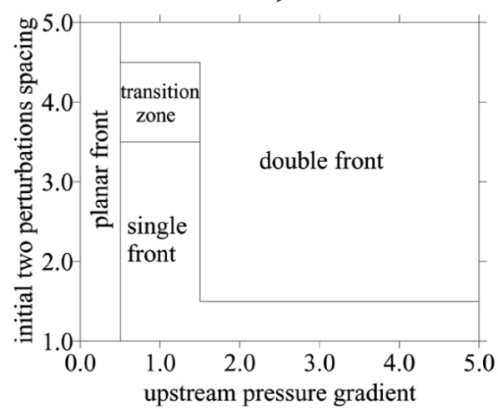

(b) $\mathrm{SS}=1.0, \quad \alpha=0.4$

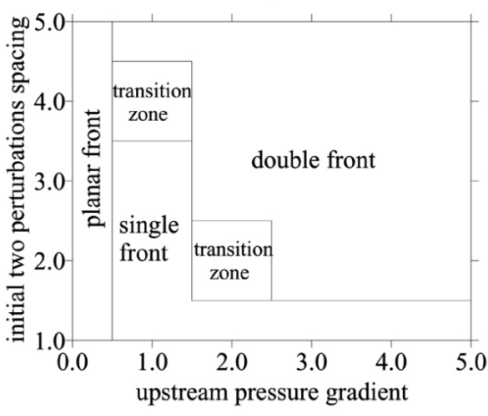

(e) $\mathrm{SS}=1.0, \quad \alpha=1.0$

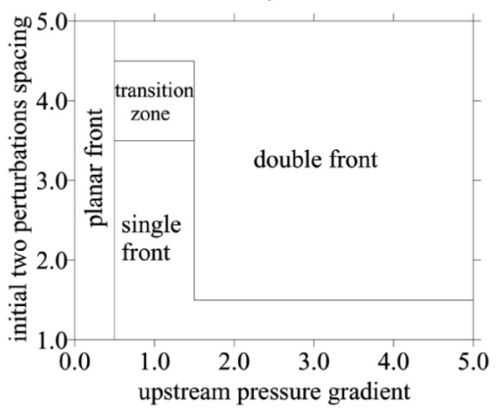

(c) $\mathrm{SS}=1.0, \alpha=0.6$

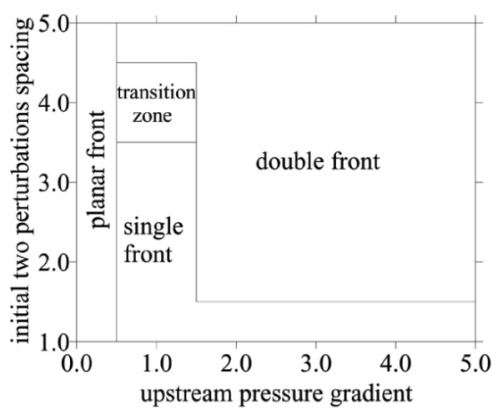

Fig. 5. Behavior diagrams for different reaction rate constants $(\alpha)$. 

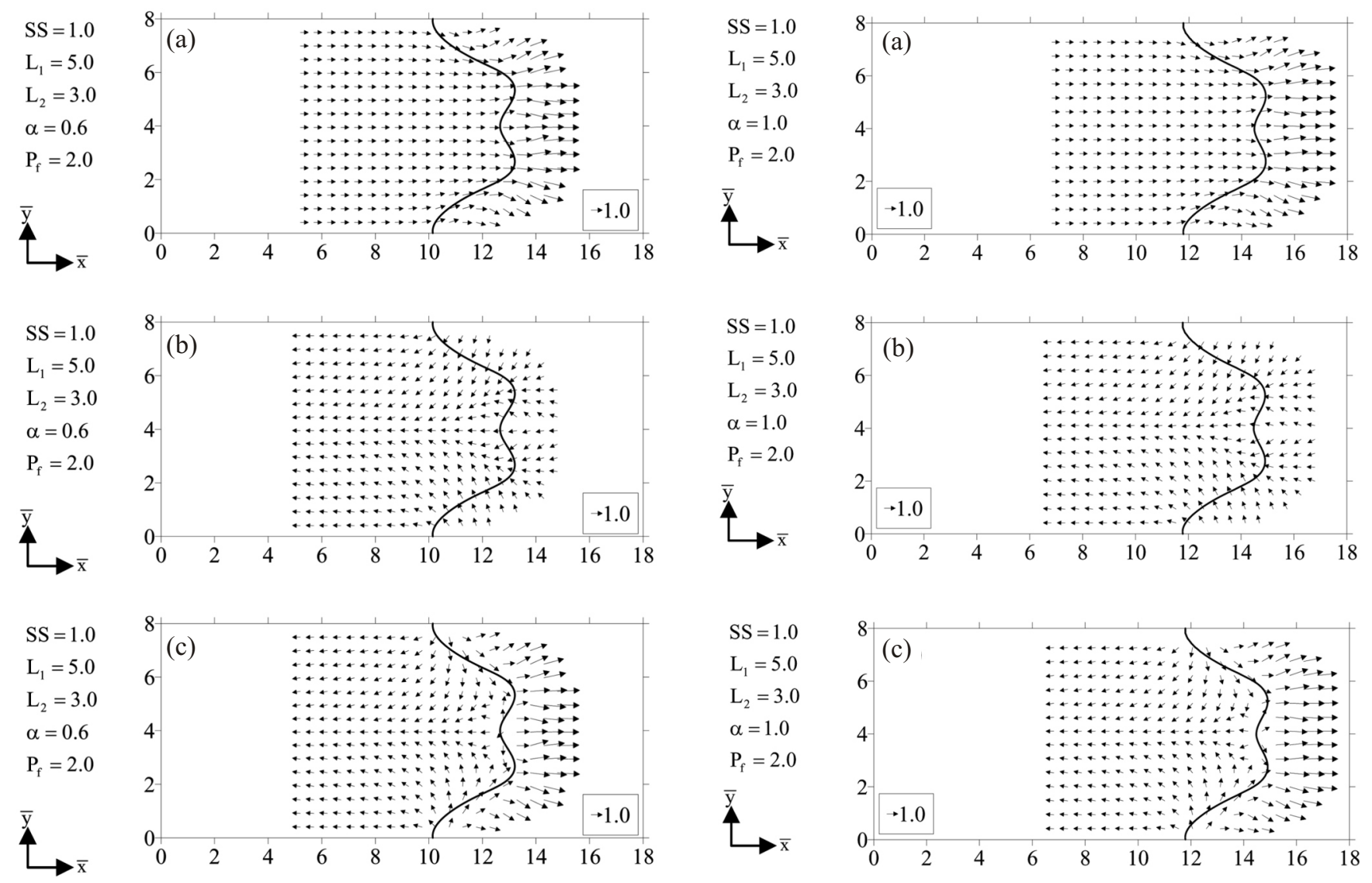

Fig. 6. (a) Advection flux; (b) diffusion flux; (c) resultant flux with $\alpha=$ 0.6 at $\tau=3.887$.

that $\alpha$ of 0.6. Therefore, the front advancing velocity increases with increasing $\alpha$ in this case. In this study, the changes of solute concentration are due to advection transport, diffusion transport and kinetic dissolution reactions. The resulting change in solute concentration due to the above processes may nonlinearly vary. Hence, front advancing velocity may not monotonically increase with $\alpha$. We herein average the simulation ending time for all sets of each $\alpha$ value except for the ones of planar front, since the simulation ending time of planar front sets all arrive at $\tau=10.0$. Figure 8 represents the average of simulation ending times for different $\alpha$. Because the simulation domain dimension is fixed, the simulation ending time is inversely proportional to the front advancing velocity. In Fig. 8 , the front advancing velocity behavior can be divided by two zones of $\alpha \leqq 0.4$ and $\alpha>0.4$ at $\alpha \leqq 0.4$, the front advancing velocity is inversely proportional to $\alpha$, whereas at $\alpha>0.4$ the front advancing velocity is positively proportional to $\alpha$ and the front advancing velocity is the slowest at $\alpha=0.4$.

The changes of dissolution reaction rate play different roles in reactive chemical transport. On the one hand, porosity increases with $\alpha$ resulting in an acceleration in front advancement, on the other hand the solute concentration of this
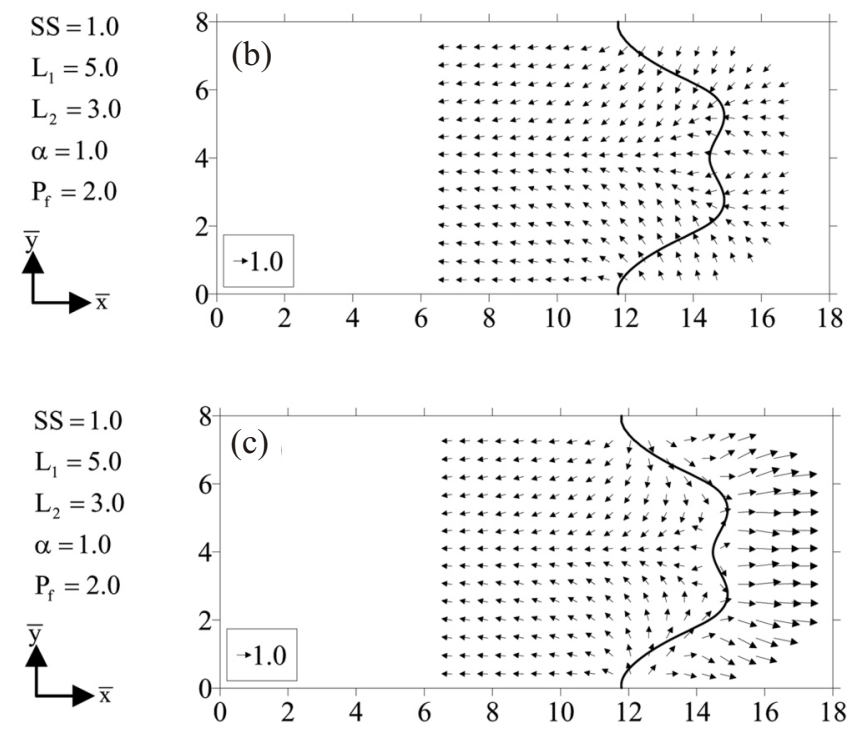

Fig. 7. (a) Advection flux; (b) diffusion flux; (c) resultant flux with $\alpha=$ 1.0 at $\tau=3.232$.

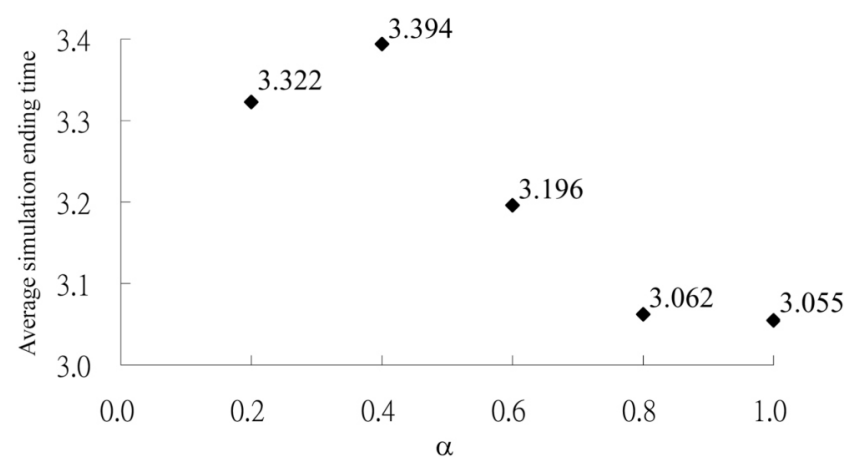

Fig. 8. Average simulation ending time for different $\alpha$.

zone may easily reach its saturated concentration, slowing down the kinetic dissolution reaction rate, and further retarding front advancement. These feedback processes are nonlinearly balancing each other, creating a complex, self-organized natural phenomenon (Ortoleva 1994).

\section{CONCLUSION}

In this study, dissolution-induced porosity change is in- 
vestigated under various combinations of upstream pressure gradient, reaction rate constant, non-uniformity spacing, and non-uniformity strength ratio. Non-uniformity strength ratio and reaction rate constant significantly affect the evolution of the reaction front. When the upstream pressure gradient is small $(<0.3)$, the two non-uniformities all develop into a planar front regardless of changing non-uniformity strength ratios, non-uniformity spacing or reaction rate constant. Moreover, the two non-uniformities tend to develop into a double-fingering front as the strength ratio increases from 0.2 to 1.0 , and into a single-fingering front as the strength ratio increases from 1.0 to 1.8. Changes in the strength ratio affect the front advancing velocity and front behavior diagram. The front advancing velocity decreases with the reaction rate constant and front advancing velocity is slowest when the reaction rate constant is equal to 0.4 . The simulated result provides a quantitative basis to analyze some natural processes of formation such as the dissolution of karst rock. Future study could consider a high flow velocity condition, and include the dispersion effect. In addition, precipitation chemical reactions, adsorption and desorption, or multiplespecies reactive chemical transport can also be incorporated into the NSPCRT model. In addition, the investigation of porosity changes induced by microbial mediation is another research area for future study.

\section{REFERENCES}

Bear, J., 1972: Dynamics of fluid in porous media. Elsevier, Amsterdam, 764 pp.

Chadam, J., D. Hoff, E. Merino, P. Ortoleva, and A. Sen, 1986: Reactive infiltration instabilities. IMA J. Appl. Math., 36, 207-221, doi: 10.1093/imamat/36.3.207. [Link]

Chen, J. S. and C. W. Liu, 2002: Numerical simulation of the evolution of aquifer porosity and species concentrations during reactive transport. Comput. Geosci., 28, 485-499, doi: 10.1016/S0098-3004(01)00084-X. [Link]

Chen, J. S. and C. W. Liu, 2004: Interaction of reactive fronts during transport in a homogeneous porous medium with initial small non-uniformity. J. Contam. Hydrol., 72, 4766, doi: 10.1016/j.jconhyd.2003.10.006. [Link]

Chen, W. and P. Ortoleva, 1990: Reaction front fingering in carbonate-cemented sandstones. Earth-Sci. Rev., 29, 183-198, doi: 10.1016/0012-8252(90)90036-U. [Link]
Daccord, G., O. Liétard, and R. Lenormand, 1993: Chemical dissolution of a porous medium by a reactive fluid-II. Convection vs reaction, behavior diagram. Chem. Eng. Sci., 48, 179-186, doi: 10.1016/0009-2509(93)80294-Z. [Link]

Emmanuel, S. and B. Berkowitz, 2005: Mixing-induced precipitation and porosity evolution in porous media. $A d v$. Water Resour., 28, 337-344, doi: 10.1016/j.advwatres. 2004.11.010. [Link]

Fredd, C. N. and H. S. Fogler, 1998: Influence of transport and reaction on wormhole formation in porous media. AIChE J., 44, 1933-1949, doi: 10.1002/aic.690440902. [Link]

Lasaga, A. C., 1998: Kinetic theory in the earth sciences. Princeton University Press, New Jersey, 811 pp.

Lerman, A., 1979: Geochemical processes. Wiley, New York, $481 \mathrm{pp}$.

Li, L., C. H. Benson, and E. M. Lawson, 2006: Modeling porosity reductions caused by mineral fouling in continuous-wall permeable reactive barriers. J. Contam. Hydrol., 83, 89-121, doi: 10.1016/j.jconhyd.2005.11.004. [Link]

Ortoleva, P. J., 1994: Geochemical Self-Organization. Clarendon Press, Oxford, 411 pp.

Ortoleva, P., E. Merino, C. Moor, and J. Chadam, 1987a: Geochemical self-organization I: Reaction-transport feedbacks and modelling approach. Am. J. Sci., 287, 979-1007.

Ortoleva, P., J. Chadam, E. Merino, and A. Sen, 1987b: Geochemical self-organization II: The reactive-infiltration instability. Am. J. Sci., 287, 1008-1040.

Renard, F., J. P. Gratier, P. Ortoleva, E. Brosse, and B. Bazin, 1998: Self-organization during reactive fluid flow in a porous medium. Geophys. Res. Lett., 25, 385-388, doi: 10.1029/97GL03781. [Link]

Singurindy, O. and B. Berkowitz, 2005: The role of fractures on coupled dissolution and precipitation patterns in carbonate rocks. Adv. Water Resour., 28, 507-521, doi: 10.1016/ j.advwatres.2005.01.002. [Link]

$\mathrm{Xu}, \mathrm{T}$. and K. Pruess, 2001: Modeling multiphase non-isothermal fluid flow and reactive geochemical transport in variably saturated fractured rocks: 1 . Methodology. Am. J. Sci., 301, 16-33, doi: 10.2475/ajs.301.1.16. [Link]

Zhao, C., B. E. Hobbs, P. Hornby, A. Ord, S. Peng, and L. Lin, 2008: Theoretical and numerical analyses of chemicaldissolution front instability in fluid-saturated porous rock. Int. J. Numer. Anal. Methods Geomech., 32, 1107-1130, doi: 10.1002/nag.661. [Link] 